\title{
Intelligent Prediction of Process Parameters for Bending Forming
}

\author{
Shengle Ren, Yinan Lai ${ }^{*}$, Guangfei Wu, Juntao Gu and Ye Dai \\ School of Mechanical and Power Engineering, Harbin University of Science and Technology, Harbin 150001, China
}

\begin{abstract}
The choice of the process parameters in the conventional tube bending forming is often based on experience and adjusted by repeated bending tests. The method of constantly testing to adjust has seriously affected the production efficiency and increased production costs. In this paper, neural network is used to establish the intelligent prediction model of the pipe forming process parameters. The obtained datum from analytical calculations, numerical simulations and experiments then serve as the training samples and test samples of neural network training. By the trained neural network, the intelligent prediction for the main process parameters including the bending moment and the boost power can be executed. The test results show that the average relative error between the simulation output and target output of bending moment and boost power is less than $2 \%$, and the predicted process parameters, i.e. bending moment and boost power, can be directly used for actual production.
\end{abstract}

Keywords: Neural network, intelligent prediction, process parameters, bending forming.

\section{INTRODUCTION}

The technology of tube bending is widely used in many fields, such as modern automobile, aerospace industry and shipbuilding, so it plays an important role in society. The shaping quality of bending tube mainly includes ellipticity, exine thinness rate and inner wall thickness rate, and there are many factors that could influence the shaping quality, such as pipe size, material, the rotating velocity of pipe die, the technological parameters in bending process, and so on. These factors have an inter-coupling relationship, so they have a non-liner and complicated influence on shaping quality of bending tube. The manipulator, lack of theoretic calculation and convenient method, always adjust and confirm technological parameters by experience and experiments when they machine different batches and type pipes with the traditional tube bender using cold bending, so the precision of shape can't be achieved by single bending forming. These results in long period, high cost, low efficiency and can't satisfy the need of rapid manufacture.

There are many researches in this field in some domestic and foreign universities. Yongjun Wang from Northwestern Polytechnical University has made an in-depth study on the intelligent control of sheet metal bending forming, established its intelligent control model by using the artificial neural network, and the technology of online prediction controlling in stretch bending process is realized [1]. His work provides a valuable reference for the intelligence of sheet metal forming. By using finite element method, Yongle Kou has investigated the influence of the pushing equipment on the wall thickness thinning and cross section ovalisation [2]. The achievements of his study lay foundations for the establishing of an accurate finite element model and the ana-

*Address correspondence to this author at the School of Mechanical and Power Engineering, Harbin University of Science and Technology, Harbin 150080, China; Tel: +86-451-86390588(office), +86-13329414010 (Mobile); Fax: +86-451-86390501; E-mails: laiyinan@hrbust.edu.cn, renshengle@163.com lyzing of the forming mechanism and quality in Aluminum alloy thin-walled tube NC bending with small bending radius; it also provides some bases for the selection of reasonable process parameters and the control of wall thickness thinning and cross section ovalisation in Aluminum alloy thin-walled tube NC bending process. Guangxiang Wang studied the influence of processing parameters on wrinkling cracking spring-back and deformation of thin-walled NC bending tube by experimentation [3]. The major reason for the extension length of mandrel influencing wrinkle and crack of tube is deeply analyzed, and the calculation formula of the most reasonable extension length of mandrel is deduced and proved. At the same time, the effects of angularity on spring-back angle and deformation of cross-section are researched, and the conclusion of the linear relationship between angularity and spring-back angle is obtained. Changping Pan from Jilin University analyzed the bending mechanism of thin-walled tube, and focused on the bending spring-back control of thin-walled tube, and analyzed and elaborated the working principle of the self-developed CNC tube bender [4]. Through the method of numerical simulation, the finite element analysis and experiment of bending process parameters on curvature stretch bending of rectangular aluminum have been achieved by Arild $\mathrm{H}$. Clausen and Odd. S. Hopperstad, et al. [5]. The researchers found that the work piece spring-back is controlled by the pretension and the strain hardening index.

Lingyi Sun researched the key technologies of neural network bending spring-back prediction [6]. He effectively solved the nonlinear mapping problem between the tension control parameters and resilience amount by establishing a kind of artificial neural network model which has been achieved by using Matlab neural network toolbox, achieved a good prediction accuracy, and verified the validity of the prediction model. A numerical wrinkling prediction system is developed for $\mathrm{NC}$ thin-walled bending process by Yang $\mathrm{He}$ [7]. The analysis of the bending process of aluminum and stainless tube has been carried out by the system. The system can be used to both predict the wrinkling phenomena and 


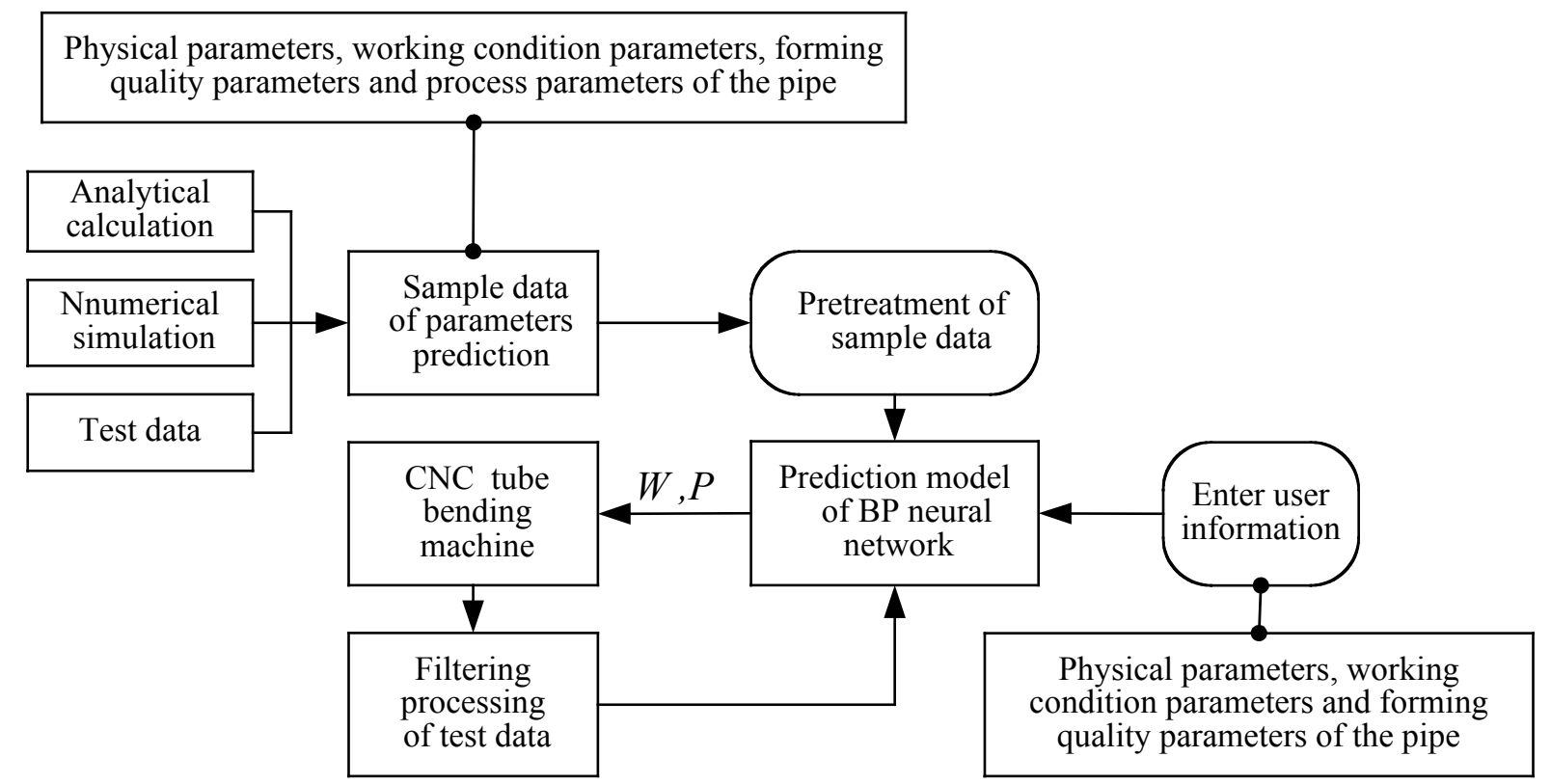

Fig. (1). Schematic drawing of prediction scheme of the tube bending forming process parameters.

analyze the bending process. Lehua Qi and Junjie Hou, who used artificial neural network method, applied 81 sets of experimental data to the neural network modeling and testing, and then established a parameters knowledge base of liquid extrusion tube and bar, which can more accurately predict the key process parameters [8]. In this paper, BP neural network has been used to predict the process parameters of tube bending, which makes the tube bending process realize intelligentization and automation, and enhance the productivity and reduce the production costs.

\section{PREDICTION OF TUBE BENDING FORMING PROCESS PARAMETERS BASED ON BP NEURAL NETWORK}

The neural network is used as the prediction model, and the obtained datum from analytical calculations, numerical simulations and experiments serve as the training samples. The process parameters database is established by the analytical calculations of the bend forming, finite element simulations and experiments. The retrieved processing parameters serve as the initial value of the predictive control. According to the different material parameters, process parameters can be dynamically adjusted by the model predictive control model so as to achieve the predictive control of the bend forming quality.

The prediction scheme of the tube bending forming process parameters for utility boiler based on BP neural network is shown in Fig. (1).

The original samples of the prediction model of process parameters are obtained by methods of analytical calculations, numerical simulations and experimental data. There are fourteen parameters in the samples, two of which, i.e. bending moment and boost power, are chosen as the goal of the network prediction. The original samples for the neural network must be pretreatment before applied to neural network. Trained neural network prediction model can be directly used in production. Users would obtain the process parameters $\mathrm{W}$ and $\mathrm{P}$ by inputting the pipe physical parameters, working parameters and objectives forming quality parameters. Then the predictive values will be sent to the tube bending machine for real-time control. The data obtained from operation by users are eventually returned as the new sample data of the neural network prediction model.

\section{ESTABLISHMENT OF THE PREDICTION MODEL OF TUBE BENDING FORMING PROCESS PARAMETERS}

BP neural network algorithm can approximate nonlinear functions with arbitrary precision, it is widely used to solve the industrial process control problem of the nonlinear characteristics and the unknown structure, and it is also suitable for building the model of multivariable nonlinear and uncertain time-varying complex system. The establishment of BP neural network model can be divided into three parts: the sample data acquisition and processing; determination of the network structure; neural network training and testing [9].

\subsection{Sample Data Acquisition and Processing}

The sample data acquisition and processing including sources of initial data samples, determination of input and output, design of the total sample network and pretreatment of input and output data.

\subsubsection{Sources of Initial Data Samples}

In the process of tube bending forming of utility boiler, the sample data of network training and testing mainly come from three ways: One is the experience data accumulated by the bending machine operators. This part of the data can also be obtained by the repeated bending test. Second one is the simulation of tube bending forming process by using the FEA software. The conditions of tube bending forming are added and changed in order to make the end results tend to the ideal of the best value. Third one is the establishment of 
bending mechanical analysis model for analytical calculation.

\subsubsection{Determination of Input and Output}

In the bending process, the processing parameters are affected by the pipe size parameters, physical parameters and the quality parameters. Hence, in the original sample the original sample, pipe outer diameter, the 12 variables of wall thickness, yield strength, elastic modulus, strength coefficient, stiffness index, bending radius, bending speed, bending angle, exine thinness rate, inner wall thickening and ellipticity are designed as network input variables, bending moment and boost power as the network output variable.

\subsubsection{Design of the Total Sample Network}

The design of the total sample determines the advantages and disadvantages of network training results. According the rule of thumb, the sample size required for the network training needs to account for 5 to 10 times of the total number of the connection weights. 320 sets of data selected from the original sample are regarded as the total sample data set of BP neural network. In the process of selecting the total sample, the balance principle of sample type has been fully considered during the sample selection. Finally, 224 sets of data were selected as the training sample set, 96 sets of data as the test sample set.

\subsubsection{Pretreatment of Input and Output Data}

The total samples must be pretreated in network training, namely the uniform normalized. Through the transform processing network's input and output data will be limited in the $[-1,1]$ or $[0,1]$ interval. BP neural network can use the Matlab toolbox built-in data to convert functions. Training samples and test samples can be normalized uniformly by using prestd function, while the input data during prediction by using trastd function, and the poststd function must be used for the reduction treatment of final predictive Output.

\subsubsection{Design of the Process Parameters Database}

The process parameters database is established by the analytical calculations of the bend forming, finite element simulations and experiments. The retrieved processing parameters serve as the initial value of the predictive control. According to the different material parameters, process parameters can be dynamically adjusted by the model predictive control model so as to achieve the predictive control of the bend forming quality, and improve the bending quality.

\subsection{Determination of the Network Structure}

In this paper, predictive control model was established by BP neural network with three hidden layers, the predictive model is shown in Fig. (2).

Input Layer: pipe outer diameter $\alpha$, wall thickness $t$, yield strength $\sigma$, elastic modulus $E$, strength coefficient $n$, stiffness index $K$, bending radius $R$, bending speed $v$, bending angle $\theta$, outer wall thinning rate $\beta$, inner wall thickening $\chi$ and ellipticity $\alpha$, a total of 12 input nodes. Output layer: moment and boost power, a total of 2 output nodes.

Hidden Layer Nodes: The role of the hidden layer node is to extract and store the internal law of the sample. The number of hidden nodes directly affects its ability of generalizing and reflecting the sample laws. Theoretically, a three-layer BP neural network can make a full approximation arbitrarily with any complex nonlinear function. Therefore, only one hidden layer is usually enough. In this paper, the number of the input network layer and output layer nodes are 12 and 2, respectively. The results showed that using a single hidden layer neural network, the network training time is very long, it is difficult to achieve the target accuracy even though the iterative steps is more than 5000. Considering the time limits in practical application, the three-hidden layer neural network can be controlled iterations within 50 steps, which greatly improved the training efficiency.

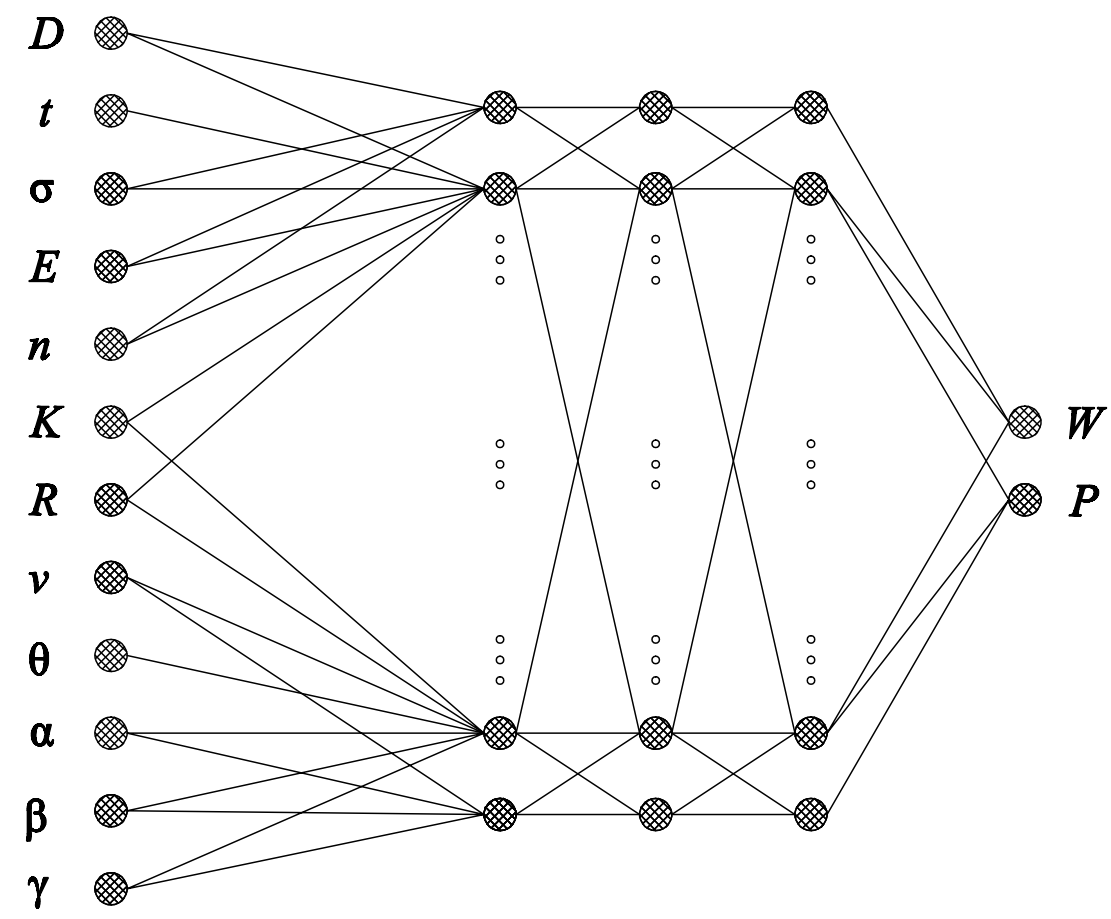

Fig. (2). Neural network prediction model of process parameters of tube bending forming. 
Firstly, the number of nodes in hidden layer has been estimated by using the empirical formula. Then through commissioning tests based on the estimated value, the best group of hidden nodes has been selected. The following is a kind of empirical formula of hidden nodes:

$m=\sqrt{n+l}+\alpha m$

Where $m$ is the number of hidden layer nodes, $n$ is input nodes, $l$ is output nodes; $\alpha$ is a constant between 1 to 10 .

According to empirical formula, taking the estimated number of hidden layer nodes as 10-10-6, and gradually increasing the number of nodes for training the same sample set, then the following results was obtained by several experiments in Table 1:

Table 1. The Results of Hidden Nodes from Several Experiments

\begin{tabular}{|c|c|c|c|}
\hline $\begin{array}{c}\text { Hidden layer } \\
\text { nodes }\end{array}$ & Training steps & Moment fitting & $\begin{array}{c}\text { Boost power } \\
\text { fitting }\end{array}$ \\
\hline \hline $10-10-6$ & 22 & 0.995 & 0.902 \\
\hline $12-12-8$ & 18 & 0.993 & 0.924 \\
\hline $16-16-8$ & 16 & 0.995 & 0.907 \\
\hline $16-15-12$ & 11 & 0.998 & 0.983 \\
\hline $18-18-15$ & 25 & 0.956 & 0.924 \\
\hline
\end{tabular}

The above table shows that hidden nodes is selected as 16-15-12, the network training convergence can be achieved just within 10 steps, and get the best fitting degree.

\subsection{BP Network Trainning and Testing}

After determining the network structure and training sample set, network training can be stated. During the training, the feedbacks of advantages and disadvantages of the network performance are used to adjust the network design. The main advantages and disadvantages of network performance are to see whether it has good generalization ability, namely the approximate capacity between the predicted results and expected results. In addition, the speed of network training for some special problems is also a basis to determine network performance. Since the input data are all more than zero, so the tansig transfer function is used in the hidden layer, the purelin transfer function is used in output layer, and trainlm function based on a LM algorithm is used in the training. The experiment results shows that target error can be achieved only within 20 iteration steps.

The partial training sample data of process parameters prediction network for utility boiler are listed in Table $\mathbf{2}$.

Final determination of the network is to constantly adjust based on the advantages and disadvantages of the training performance, and when the test results of test samples meet the expectations, then it can be say that the form of network was finally established.

\section{EXAMINATIONS AND ANALYSIS OF RESULTS}

According to the process of network training above, it can be found that the network training is a kind of process in which a output result constantly feedback to adjust the network training. Then, the final qualified BP neural network must satisfy the desired effect. Otherwise, the network is still unsatisfactory and requires further improvement. The bending moment and boost power as the network output must meet the requirements of actual production, i.e. errors between predictive value and expectation value need to be controlled within $2 \%$, that is, the relative error must be controlled below $2 \%$. In addition, the training speed must be fast. Neural network training error gradient is shown in Fig. (3).

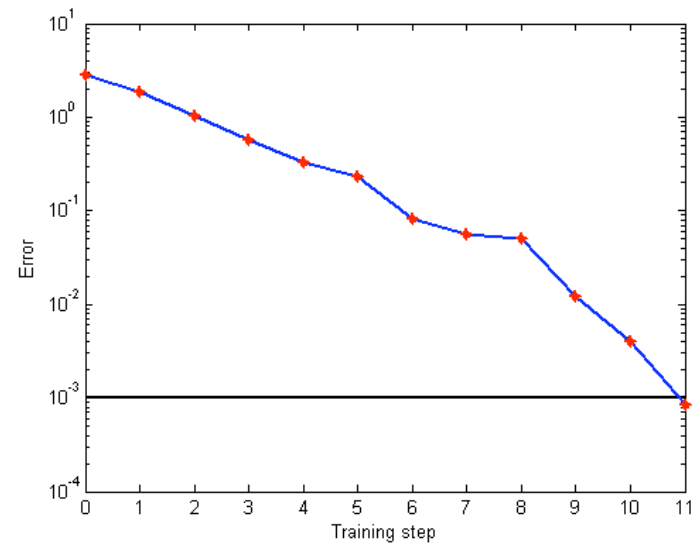

Fig. (3). Schematic drawing of neural network training error gradient.

Table 2. Partial Training Sample Data of Process Parameters Prediction Network for Utility Boiler

\begin{tabular}{|c|c|c|c|c|c|c|c|c|c|c|c|c|c|}
\hline \multicolumn{12}{|c|}{ Network input data } & \multicolumn{2}{|c|}{ Target output } \\
\hline$D$ & $t$ & $\sigma$ & $E$ & $n$ & $\boldsymbol{K}$ & $\boldsymbol{R}$ & $v$ & $\theta$ & $\alpha \%$ & $\beta \%$ & $x \%$ & $W$ & $\boldsymbol{P}$ \\
\hline 63 & 7 & 310 & 211 & 0.3 & 390 & 130 & 0.5 & 90 & 4.28 & 10.55 & 13.67 & 1.008 & 4.9 \\
\hline 63 & 7 & 318 & 217 & 0.31 & 380 & 130 & 0.5 & 90 & 4.25 & 10.52 & 13.68 & 1.009 & 4.8 \\
\hline 63 & 11 & 315 & 225 & 0.26 & 386 & 130 & 0.5 & 90 & 2.5 & 10.6 & 12.66 & 1.562 & 5.5 \\
\hline 63 & 11 & 309 & 213 & 0.34 & 392 & 130 & 0.5 & 90 & 2.473 & 10.55 & 12.65 & 1.563 & 5.5 \\
\hline 54 & 7 & 310 & 217 & 0.34 & 396 & 118 & 0.5 & 90 & 3.49 & 9.8 & 12.67 & 1.038 & 5.3 \\
\hline 54 & 7 & 312 & 214 & 0.29 & 406 & 118 & 0.5 & 90 & 3.48 & 9.77 & 12.68 & 1.025 & 5.2 \\
\hline 54 & 4 & 310 & 217 & 0.27 & 396 & 130 & 0.5 & 180 & 5.95 & 8.81 & 12.84 & 0.574 & 3.9 \\
\hline 54 & 4 & 314 & 203 & 0.34 & 402 & 130 & 0.5 & 180 & 5.82 & 8.72 & 12.9 & 0.567 & 4.0 \\
\hline
\end{tabular}


Table 3. Comparison of the Simulation Output and Target Output of the Bending Moment $W$

\begin{tabular}{|c|c|c|c|c|c|c|}
\hline Target output $\boldsymbol{W}$ & \multicolumn{4}{|c|}{ Simulation output $\boldsymbol{W}$} & Standard deviation & Maximum relative error (\%) \\
\hline \hline 0.671 & 0.6698 & 0.6682 & 0.6672 & 0.6731 & 0.0026 & -0.57 \\
\hline 0.645 & 0.6442 & 0.6394 & 0.6464 & 0.6386 & 0.0038 & -0.99 \\
\hline 1.138 & 1.1369 & 1.1395 & 1.1391 & 1.1388 & 0.0012 & 0.13 \\
\hline 1.025 & 1.0239 & 1.0261 & 1.0236 & 1.0195 & 0.0028 & -0.53 \\
\hline 1.681 & 1.6832 & 1.6792 & 1.6831 & 1.6792 & 0.0023 & 0.13 \\
\hline
\end{tabular}

Table 4. Comparison of the Simulation Output and Target Output of the Boost Power $P$

\begin{tabular}{|c|c|c|c|c|c|c|}
\hline Target output $\boldsymbol{P}$ & \multicolumn{4}{|c|}{ Simulation output $\boldsymbol{P}$} & Standard deviation & Maximum relative error (\%) \\
\hline \hline 4.6 & 4.6138 & 4.6154 & 4.5347 & 4.6491 & 0.0485 & -1.42 \\
\hline 4.3 & 4.2764 & 4.3156 & 4.4012 & 4.3235 & 0.0522 & -1.24 \\
\hline 6.7 & 6.6837 & 6.6378 & 6.6714 & 6.7154 & 0.0321 & -0.93 \\
\hline 5.8 & 5.7645 & 5.9010 & 5.8226 & 5.7761 & 0.0712 & 1.74 \\
\hline 7.1 & 7.1843 & 7.0347 & 7.1352 & 7.2130 & 0.0783 & 1.59 \\
\hline
\end{tabular}

From the Fig. (3), it can be seen that the accuracy of the network training has reached 0.00083405 in the 11 -step, the precision of target error is 0.001 , and hence both of the training speed and training accuracy achieved the intended target. Partial results of simulation output and target output of test samples for the bending moment and boost power are listed in Table $\mathbf{3}$ and Table $\mathbf{4}$, which are selected from 4-time consecutive network prediction.

The linear fitting charts of moment linear and boost power of network input and target output of the test sample are shown in Fig. (4) and Fig. (5) respectively.

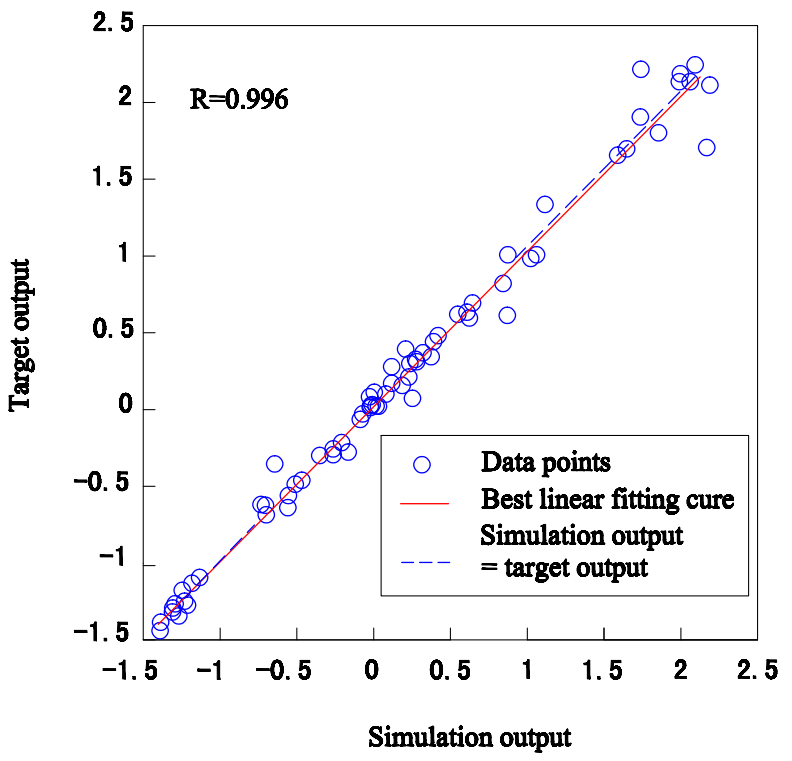

Fig. (4). Moment linear fitting chart.

According to the linear fitting charts of moment linear and boost power above, it can be seen that the correlation coefficient $\mathrm{R}$ reflecting the fitting degree is 0.996 and 0.983 respectively, very close to 1 . The predictive value and expected value are in high fitting degree. Through the 4-time consecutive network tests, the maximum relative errors between the simulation output and the target output are all controlled within $2 \%$, which meets the accuracy requirement of this case and confirms the reliability and validity of BP neural network technology for the tube bending process parameters prediction.

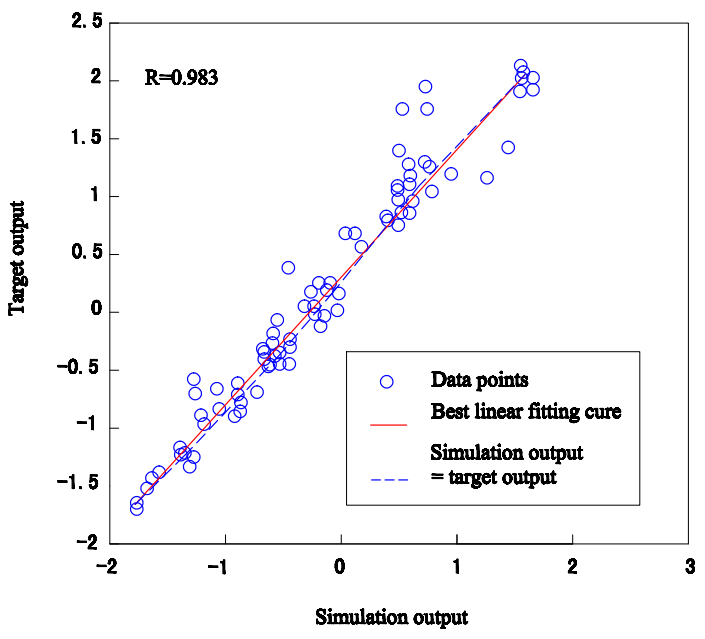

Fig. (5). Boost power linear fitting chart.

\section{CONCLUSIONS}

There are many factors that could influence the shaping quality, and the relationship between these factors is intercoupling and nonlinear. Hence, the determination of process parameters by continuously adjusting the parameters often takes a lot of time and increases the production cost in the actual production of tube bending. In this paper, BP neural network is used to establish the intelligent prediction model 
of the pipe forming process parameters, and the main process parameters including the bending moment and the boost power were predicted. The main results are as follows:

(1) The prediction scheme of the tube bending forming process parameters for utility boiler was established based on BP neural network, the moment and boost power network serve as the prediction targets.

(2) The BP Neural network prediction model was established, and the network structure, network training and testing methods were designed.

(3) The test results show that the prediction error of the bending moment and the boost power can be maintained at below $2 \%$, fully meet the required precision of tube bending. The trained neural network model can be directly used to guide the production practice.

\section{ACKNOWLEDGMENTS}

The research is supported by Project of ChineseBulgarian Scientific and Technological Cooperation (2009DFA71860), National Science Foundation of Heilongjiang Province (ZJG2007-02-02), Program for New Century Excellent Talents in Heilongjiang Provincial University (1153-NCET-005) and National Science Youth Foundation of Heilongjiang Province (QC2009C50).

\section{REFERENCES}

[1] Y. J. Wang, Study on Intelligent Control of Precise Stretch Bending Forming, D.S. thesis, Northwestern Polytechnical University, Xian, China, 2005

[2] Y. L. Kou, Experimental Research on NC Bending with Small Bending Radius of Aluminum Alloy Thin-Walled Tube, Northwestern Polytechnical University, Xian, China, 2007.

[3] G. X. Wang, H. Yang, H. Li, M. Zhan, and R. J. Gu, Experimental Study of the Influence of Processing Parameters on Forming Quality of Thin-Walled NC Bending Tube, Mech. Sci. Tech., vol. 24, pp. 995-998, August 2005.

[4] Ch. P. Pan, Research on the Bending Spring-Back of Thin-Walled Tube and the Control System of Tube Bending Machine, Jilin University, Changchun, China, 2008.

[5] A. H. Clausen, O. S. Hopperstad, and M. Langseth, "Sensitivity of model parameters in stretch bending of aluminum extrusions", Int. J. Mech. Sci., vol. 43, pp. 427-453, February 2001.

[6] L. Y. Sun, Y. J. Wang, and J. B. Wang, " Springback prediction of stretch bending using artificial neural network", Mech. Sci. Tech., vol. 27, pp. 65-68, January 2008.

[7] Y. He, and L. Yan, "Wrinkling analysis for forming limit of tube bending processes", J. Mater. Process. Technol., vol. 152, pp. 363369, October 2004.

[8] L. H. Qi, J. J. Hou, F. Yang, and H. J. Li, "Establishing the knowledge base of parameters for shaping tube and bar products on the neural network", Acta Aeronautica Et Astronautica Sinica, vol. 19, pp. 744-747, November 1998.

[9] Y. W. Li, Research and Implement Intelligent Forecast System Based on ANN, North China University of Technology, Beijing, China, 2006. 